\title{
THE INVISCID LIMIT OF THE MODIFIED BENJAMIN-ONO-BURGERS EQUATION
}

\author{
HUA ZHANG ${ }^{\otimes}$ and YUQIN KE
}

(Received 23 June 2010)

\begin{abstract}
We prove that the modified Benjamin-Ono-Burgers equation is globally well-posed in $H^{s}$ for $s>0$. Moreover, we show that the solution of the modified Benjamin-Ono-Burgers equation converges to that of the modified Benjamin-Ono equation in the natural space $C\left([0, T] ; H^{s}\right), s \geq 1 / 2$, as the dissipative coefficient $\epsilon$ goes to zero, provided that the $L^{2}$ norm of the initial data is sufficiently small.
\end{abstract}

2010 Mathematics subject classification: primary 35Q53; secondary 49K40.

Keywords and phrases: modified Benjamin-Ono-Burgers equation, global well-posedness, inviscid limit.

\section{Introduction}

The purpose of this paper is to study the global well-posedness and the inviscid limit behaviour of the Cauchy problem for the modified Benjamin-Ono-Burgers (mBOB) equation

$$
\begin{gathered}
u_{t}+\mathcal{H} u_{x x}-\epsilon u_{x x}=u^{2} u_{x}, \\
u(x, 0)=\phi(x),
\end{gathered}
$$

where $u(x, t): \mathbb{R} \times \mathbb{R} \rightarrow \mathbb{R}, 0<\epsilon \leq 1$ and $\mathcal{H}$ is the Hilbert transform:

$$
\mathcal{H} u(x)=\frac{1}{\pi} \text { p.v. } \int_{-\infty}^{+\infty} \frac{u(y)}{x-y} d y .
$$

When the nonlinearity in (1.1) is $-u^{2} u_{x}$, it can also be treated by our method.

Formally, letting $\epsilon=0$, then (1.1) becomes the modified Benjamin-Ono (mBO) equation:

$$
u_{t}+\mathcal{H} u_{x x}=u^{2} u_{x}, \quad u(x, 0)=\phi(x) .
$$

Thus it is natural to conjecture that the solution of (1.1) converges to that of (1.3) as $\epsilon$ tends to zero in the natural space $C\left([0, T]: H^{s}\right)$. The same problem for the BenjaminOno-Burgers equation (with quadratic nonlinearity $u u_{x}$ in (1.1)) was suggested by

Zhang was partially supported by the Science Research Startup Foundation of North China University of Technology.

(C) 2010 Australian Mathematical Publishing Association Inc. 0004-9727/2010 \$16.00 
Tao [13], who proved that the Benjamin-Ono equation is globally well-posed in $H^{1}(\mathbb{R})$. The inviscid limit problems are very interesting from the physical viewpoint and have been studied by many authors $[5,15,16]$. The limit in the low regularity space was first studied by Guo and Wang [5] where they used the $l^{1}$-type $X^{s, b}$ structure.

In [2], Guo showed that (1.3) is globally well-posed for $\phi \in H^{s}, s \geq 1 / 2$, and $\|\phi\|_{L^{2}}$ sufficiently small. In this paper, we show that (1.1) is globally well-posed for $\phi \in H^{s}$, $s>0$. In [14], Vento considered the Cauchy problem for dissipative Benjamin-Ono equations

$$
\begin{gathered}
u_{t}+\mathcal{H} u_{x x}+|D|^{\alpha} u+u u_{x}=0, \quad t>0, x \in \mathbb{R}, \\
u(x, 0)=\phi(x),
\end{gathered}
$$

where $|D|^{\alpha}$ is the Fourier multiplier with symbol $|\xi|^{\alpha}, 0<\alpha \leq 2$. When $0 \leq \alpha<1$, the author gave the ill-posedness in $H^{s}(\mathbb{R}), s \in \mathbb{R}$, in the sense that the flow map $u_{0} \mapsto u$ (if it exists) fails to be $\mathbb{C}^{2}$ at the origin. For $1<\alpha \leq 2$, the author proved the global well-posedness in $H^{s}(\mathbb{R}), s>-\alpha / 4$. Comparing to [14], we mainly consider the situation $\alpha=2$ and with nonlinearity $-u^{2} u_{x}$. In [3], Guo considered the Cauchy problem for the dispersion generalized Benjamin-Ono equation

$$
\begin{gathered}
\partial_{t} u+|D|^{1+\alpha} \partial_{x} u+u u_{x}=0, \quad(x, t) \in \mathbb{R}^{2}, \\
u(x, 0)=u_{0}(x),
\end{gathered}
$$

where $0 \leq \alpha \leq 1$, and showed that (1.5) is locally well-posed in $H^{s}$ for $s>1-\alpha$. The $\alpha=0$ result of [3] follows from our estimates.

The main ingredients of our ideas are the methods in [5] combined with the new observation in [2] for the modified Benjamin-Ono equation. However, there are some new difficulties, since the resolution spaces are different from the one used in [5]. Fortunately, we can overcome these difficulties by using the ideas from $[5,6,10]$ and some new techniques.

We now give some notation. Let $\eta_{0}: \mathbb{R} \rightarrow[0,1]$ denote an even smooth function supported in $[-8 / 5,8 / 5]$ and equal to 1 in $[-5 / 4,5 / 4]$. For $k \in \mathbb{Z}$, let $\chi_{k}(\xi)=$ $\eta_{0}\left(\xi / 2^{k}\right)-\eta_{0}\left(\xi / 2^{k-1}\right)$, where $\chi_{k}$ is supported in $\left\{\xi:|\xi| \in\left[(5 / 8) \cdot 2^{k},(8 / 5) \cdot 2^{k}\right]\right\}$ and

$$
\chi_{\left[k_{1}, k_{2}\right]}=\sum_{k=k_{1}}^{k_{2}} \chi_{k} \quad \text { for any } k_{1} \leq k_{2} \in \mathbb{Z} .
$$

For simplicity of notation, let $\eta_{k}=\chi_{k}$ if $k \geq 1$ and $\eta_{k} \equiv 0$ if $k \leq-1$. For $k_{1} \leq k_{2} \in \mathbb{Z}$, let

$$
\eta_{\left[k_{1}, k_{2}\right]}=\sum_{k=k_{1}}^{k_{2}} \eta_{k} \quad \text { and } \quad \eta_{\leq k_{2}}=\sum_{k=-\infty}^{k_{2}} \eta_{k} .
$$

For $k \in \mathbb{Z}$, let $P_{k}$ denote the operators on $L^{2}(\mathbb{R})$ defined by

$$
\widehat{P_{k} u}(\xi)=\chi_{k}(\xi) \widehat{u}(\xi) .
$$


By a slight abuse of notation, we also define the operators $P_{k}$ on $L^{2}(\mathbb{R} \times \mathbb{R})$ by formulas $\mathcal{F}\left(P_{k} u\right)(\xi, \tau)=\chi_{k}(\xi) \mathcal{F}(u)(\xi, \tau)$. For $l \in \mathbb{Z}$, let

$$
P_{\leq l}=\sum_{k \leq l} P_{k}, \quad P_{\geq l}=\sum_{k \geq l} P_{k} .
$$

For $\xi \in \mathbb{R}$, let $\omega(\xi)=-|\xi| \xi$. For $k \in \mathbb{Z}$, let $I_{k}=\left\{\xi:|\xi| \in\left[2^{k-1}, 2^{k+1}\right]\right\}$. For $k \in \mathbb{Z}_{+}$, let $\widetilde{I}_{k}=[-2,2]$ if $k=0$ and $\widetilde{I}_{k}=I_{k}$ if $k \geq 1$. For $k \in \mathbb{Z}_{+}$and $j \geq 0$, let

$$
D_{k, j}=\left\{(\xi, \tau) \in \mathbb{R} \times \mathbb{R}: \xi \in \widetilde{I}_{k}, \tau-\omega(\xi) \in \widetilde{I}_{j}\right\} .
$$

We introduce the space used in [2,6]. First we define the $X^{s, b}$-type Banach spaces $X_{k}(\mathbb{R} \times \mathbb{R})$ for $k \in \mathbb{Z}_{+}$as follows:

$$
\begin{aligned}
X_{k}=\left\{f \in L^{2}\left(\mathbb{R}^{2}\right): f \text { is supported in } \widetilde{I}_{k} \times \mathbb{R}\right. \text { and } \\
\left.\|f\|_{X_{k}}:=\sum_{j=0}^{\infty} 2^{j / 2} \beta_{k, j}\left\|\eta_{j}(\tau-w(\xi)) \cdot f(\xi, \tau)\right\|_{L_{\xi, \tau}^{2}}<\infty\right\},
\end{aligned}
$$

where

$$
\beta_{k, j}=1+2^{2(j-2 k) / 5}
$$

The coefficients $\beta_{k, j}$ are chosen to guarantee the trilinear estimates so that Lemma 4.1 holds. For $k \geq 100$, we also define the Banach spaces $Y_{k}=Y_{k}\left(\mathbb{R}^{2}\right)$ :

$$
\begin{aligned}
Y_{k}=\left\{f \in L^{2}\left(\mathbb{R}^{2}\right): f \text { is supported in } \bigcup_{j=0}^{k-1} D_{k, j}\right. \text { and } \\
\left.\quad\|f\|_{Y_{k}}:=2^{-k / 2}\left\|\mathcal{F}^{-1}[(\tau-\omega(\xi)+i) f(\xi, \tau)]\right\|_{L_{x}^{1} L_{t}^{2}}<\infty\right\} .
\end{aligned}
$$

Then for $k \in \mathbb{Z}_{+}$, we define

$$
Z_{k}:=X_{k} \text { if } k \leq 99 \text { and } Z_{k}:=X_{k}+Y_{k} \text { if } k \geq 100 .
$$

The spaces $Z_{k}$ are our basic Banach spaces.

For $s \geq 0$, we define the Banach spaces $F^{s}=F^{s}(\mathbb{R} \times \mathbb{R})$,

$$
F^{s}=\left\{u \in \mathcal{S}^{\prime}(\mathbb{R} \times \mathbb{R}):\|u\|_{F^{s}}^{2}=\sum_{k=0}^{\infty} 2^{2 s k}\left\|\eta_{k}(\xi) \mathcal{F}(u)\right\|_{Z_{k}}^{2}<\infty\right\} ;
$$

and $N^{s}=N^{s}(\mathbb{R} \times \mathbb{R})$,

$$
\begin{aligned}
N^{s}=\{u & \in \mathcal{S}^{\prime}(\mathbb{R} \times \mathbb{R}):\|u\|_{N^{s}}^{2} \\
& \left.=\sum_{k=0}^{\infty} 2^{2 s k}\left\|\eta_{k}(\xi)(\tau-\omega(\xi)+i)^{-1} \mathcal{F}(u)\right\|_{Z_{k}}^{2}<\infty\right\} .
\end{aligned}
$$


For $T \geq 0$, we define the time-localized spaces $F^{s}(T)$ and $N^{s}(T)$ by

$$
\begin{aligned}
\|u\|_{F^{s}(T)} & =\inf _{w \in F^{s}}\left\{\|w\|_{F^{s}}, w(t)=u(t) \text { on }[0, T]\right\}, \\
\|u\|_{N^{s}(T)} & =\inf _{w \in N^{s}}\left\{\|w\|_{N^{s}}, w(t)=u(t) \text { on }[0, T]\right\} .
\end{aligned}
$$

For $\phi \in L^{2}(\mathbb{R})$, we denote by $W_{0}$ the semigroup associated with the $\mathrm{mBO}$ equation

$$
\mathcal{F}_{x}\left(W_{0}(t) \phi\right)(\xi)=\exp [i \omega(\xi) t] \widehat{\phi}(\xi), \quad \forall t \in \mathbb{R}, \phi \in \mathcal{S}^{\prime} .
$$

For $0<\epsilon \leq 1$, we denote by $W_{\epsilon}$ the semigroup associated with the free evolution of (1.1),

$$
\mathcal{F}_{x}\left(W_{\epsilon}(t) \phi\right)(\xi)=\exp \left[-\epsilon \xi^{2} t+i \xi|\xi| t\right] \widehat{\phi}(\xi), \quad \forall t \geq 0, \phi \in \mathcal{S}^{\prime}
$$

We extend $W_{\epsilon}$ to a linear operator defined on the whole real axis by setting

$$
\mathcal{F}_{x}\left(W_{\epsilon}(t) \phi\right)(\xi)=\exp \left[-\epsilon \xi^{2}|t|+i \xi|\xi| t\right] \widehat{\phi}(\xi), \quad \forall t \in \mathbb{R}, \phi \in \mathcal{S}^{\prime} .
$$

To study the low regularity of (1.1), we introduce a variant version of Bourgain's space with dissipation

$$
\|u\|_{X^{b, s, 2}}=\left\|\left\langle i(\tau-\omega(\xi))+|\xi|^{2}\right\rangle^{b}\langle\xi\rangle^{s} \widehat{u}\right\|_{L^{2}\left(\mathbb{R}^{2}\right)},
$$

where $\langle\cdot\rangle=\left(1+|\cdot|^{2}\right)^{1 / 2}$. The time-localized spaces is similar to (1.12). This type of space was introduced by Molinet and Ribaud in [9]. The standard $X^{b, s}$ space used by Bourgain [1] and Kenig et al. [7] is defined by

$$
\|u\|_{X^{b, s}}=\left\|\langle\tau-\omega(\xi)\rangle^{b}\langle\xi\rangle^{s} \widehat{u}\right\|_{L^{2}\left(\mathbb{R}^{2}\right)} .
$$

The space $X^{1 / 2, s, 2}$ turns out to be very useful for capturing both dispersive and dissipative effects. For global well-posedness, we follow the methods of Molinet and Ribaud [9], by using $X^{b, s}$-type space combined with the dissipative structures. Similar results were obtained by Vento [14] for the Benjamin-Ono-Burgers equation (with nonlinearity $u u_{x}$ in (1.1)).

Theorem 1.1. Assume that $0<\epsilon \leq 1, s>0$ and $\phi \in H^{s}(\mathbb{R})$. For any $T>0$, there exists a unique solution $u_{\epsilon}$ of (1.1) in

$$
Z_{T}=C\left([0, T], H^{s}\right) \cap X_{T}^{1 / 2, s, 2} .
$$

Moreover, the solution map $\Phi_{T}^{\epsilon}: \phi \rightarrow u$ is smooth from $H^{s}(\mathbb{R})$ to $Z_{T}$ and $u$ belongs to $C\left((0, \infty), H^{\infty}(\mathbb{R})\right)$.

We show the uniform global well-posedness for Equation (1.1) with respect to $\epsilon$.

THEOREM 1.2. Assume that $\phi \in H^{1 / 2}, 0<\epsilon \leq 1$ and $\|\phi\|_{2} \ll 1$.

(a) Existence. For any $T>0$, there exists a solution $u$ to the Cauchy problem (1.1) satisfying

$$
u \in F^{1 / 2}(T) \subset C\left([-T, T]: H^{1 / 2}\right) .
$$


(b) Uniqueness. The solution mapping $\Phi_{T}^{\epsilon}: \phi \rightarrow u$ is the unique continuous extension of the classical solution $H^{\infty} \rightarrow C\left([-T, T]: H^{\infty}\right)$.

(c) Lipschitz continuity. For any $R>0$, the mapping $\Phi_{T}^{\epsilon}: \phi \rightarrow u$ is Lipschitz continuous from $\left\{\phi \in H^{1 / 2}:\|\phi\|_{H^{1 / 2}}<R,\|\phi\|_{L^{2}} \ll 1\right\}$ to $C\left([-T, T]: H^{1 / 2}\right)$.

(d) Persistence of regularity. If in addition $\phi \in H^{s}$ for some $s>1 / 2$, then the solution u belongs to $H^{S}$.

For the limit behaviour, we have the following theorem.

THEOREM 1.3. Assume that $\phi \in H^{1 / 2}$ and $\|\phi\|_{2} \ll 1$. Then, for any $T>0$, the solution of (1.1) obtained in Theorem 1.2 converges to that of $(1.3)$ in $C\left([0, T] ; H^{s}\right)$ for $s \geq 1 / 2$ if $\epsilon$ goes to 0 .

In Sections 2-4 we give the proofs of Theorems 1.1-1.3.

\section{Proof of Theorem 1.1}

In this section we prove Theorem 1.1. Comparing the procedure of [14, Section 4], we can easily obtain Theorem 1.1 if the proposition below holds. In particular, the proof that $u$ belongs to $C\left((0, \infty), H^{\infty}(\mathbb{R})\right)$ is parallel to the proof in Section 4 in [14], and so we omit it.

PROPOSITION 2.1. Let $s>0,0<\eta \ll 1$; then there exists $C_{s, \eta}>0$ such that, for any $u_{1}, u_{2}, u_{3}$ on $\mathbb{R} \times \mathbb{R}$,

$$
\left\|\partial_{x}\left(u_{1} u_{2} u_{3}\right)\right\|_{X^{-1 / 2+\eta, s, 2}} \leq C\left\|u_{1}\right\|_{X^{1 / 2, s, 2}}\left\|u_{2}\right\|_{X^{1 / 2, s, 2}}\left\|u_{3}\right\|_{X^{1 / 2, s, 2}} .
$$

We now utilize Tao's $[k ; Z]$-multiplier from [12] to prove Proposition 2.1. For simplicity, We review some notation Tao used in [12]. We use $A \lesssim B$ to denote the statement that $A \leq C B$ for some large constant $C$ which may vary from line to line and depend on various parameters such as the dimension $n$, and we use $A \sim B$ to denote the statement that $A \lesssim B \lesssim A$. Let $Z$ be any abelian additive group with an invariant measure $d \xi$. For any integer $k \geq 2$, we let $\Gamma_{k}(Z)$ denote the hyperplane

$$
\Gamma_{k}(Z):=\left\{\left(\xi_{1}, \ldots, \xi_{k}\right) \in Z^{k}: \xi_{1}+\cdots+\xi_{k}=0\right\},
$$

which is endowed with the measure

$$
\int_{\Gamma_{k}(Z)} f:=\int_{Z^{k-1}} f\left(\xi_{1}, \ldots, \xi_{k-1},-\xi_{1}-\cdots-\xi_{k-1}\right) d \xi_{1} \cdots d \xi_{k-1} .
$$

$\mathrm{A}[k ; Z]$-multiplier is defined to be any function $m: \Gamma_{k}(Z) \rightarrow \mathbb{C}$, and the multiplier norm $\|m\|_{[k ; Z]}$ is defined to be the best constant such that the inequality

$$
\left|\int_{\Gamma_{k}(Z)} m(\xi) \prod_{j=1}^{k} f_{j}\left(\xi_{j}\right)\right| \leq\|m\|_{[k ; Z]} \prod_{j=1}^{k}\left\|f_{j}\right\|_{L^{2}(Z)}
$$

holds for all test functions $f_{j}$ on $Z$. For given $\tau, \xi$ and $h(\cdot)$, we write

$$
\lambda:=\tau-h(\xi) \text {. }
$$


Similarly, we put $\lambda_{j}:=\tau_{j}-h_{j}\left(\xi_{j}\right)$. The quantities $N_{j}$ and $L_{j}$ measure the spatial frequency of the $j$ th wave and how it resembles a free solution respectively, while the quantity $H$ measures the amount of resonance. In this paper, we consider

$$
h(\xi)=-\xi_{1}\left|\xi_{1}\right|-\xi_{2}\left|\xi_{2}\right|-\xi_{3}\left|\xi_{3}\right|=-\lambda_{1}-\lambda_{2}-\lambda_{3},
$$

which measures the extent to which the spatial frequencies $\xi_{1}, \xi_{2}, \xi_{3}$ can resonate with each other. By dyadic decomposition of the variables $\xi_{j}, \lambda_{j}$, as well as the function $h(\xi)$, one is led to consider

$$
\left\|X_{N_{1}, N_{2}, N_{3} ; H ; L_{1}, L_{2}, L_{3}}\right\|_{[3, \mathbb{R} \times \mathbb{R}]},
$$

where $X_{N_{1}, N_{2}, N_{3} ; H ; L_{1}, L_{2}, L_{3}}$ is the following multiplier:

$$
X_{N_{1}, N_{2}, N_{3} ; H ; L_{1}, L_{2}, L_{3}}(\xi, \tau):=\chi|h(\xi)| \sim H \prod_{j=1}^{3} \chi\left|\xi_{j}\right| \sim N_{j} \chi_{\left|\lambda_{j}\right| \sim L_{j}} .
$$

Define the quantities $N_{\max } \geq N_{\text {med }} \geq N_{\min }$ to be the maximum, median, and minimum of $N_{1}, N_{2}, N_{3}$ respectively. $L_{\max } \geq L_{\text {med }} \geq L_{\min }$ are similar. In this paper, we always assume that $N_{j}, L_{j}$ are dyadic numbers. From the identities $\xi_{1}+\xi_{2}+\xi_{3}=0$ and $\lambda_{1}+\lambda_{2}+\lambda_{3}+h(\xi)=0$ on the support of the multiplier, we see that $X_{N_{1}, N_{2}, N_{3} ; H ; L_{1}, L_{2}, L_{3}}$ vanishes unless

$$
N_{\text {max }} \sim N_{\text {med }} \quad \text { and } \quad L_{\max } \sim \max \left(H, L_{\text {med }}\right) .
$$

From the estimate in [6],

$$
|H| \sim|\xi|_{\max } \cdot|\xi|_{\min }
$$

where

$$
\sum_{j=1}^{3} \xi_{j}=0, \quad|\xi|_{\max }=\max \left(\left|\xi_{1}\right|,\left|\xi_{2}\right|,\left|\xi_{3}\right|\right)
$$

and

$$
|\xi|_{\min }=\min \left(\left|\xi_{1}\right|,\left|\xi_{2}\right|,\left|\xi_{3}\right|\right)
$$

Lemma 2.2 [3, Lemma 4.3]. Let $H, N_{1}, N_{2}, N_{3}, L_{1}, L_{2}, L_{3}>0$ obey (2.2) and (2.3).

Then:

(i) if $N_{\max } \sim N_{\min }$ and $L_{\max } \sim N_{\max } N_{\min }$, then

$$
\text { (2.1) } \lesssim L_{\text {min }}^{1 / 2} L_{\text {med }}^{1 / 4} \text {; }
$$

(ii) if $N_{2} \sim N_{3} \gg N_{1}$ and $N_{\max } N_{\min } \sim L_{1} \gtrsim L_{2}, L_{3}$, then

$$
(2.1) \lesssim L_{\min }^{1 / 2} N_{\max }^{-1 / 2} \min \left(N_{\max } N_{\min }, \frac{N_{\max }}{N_{\min }} L_{\text {med }}\right)^{1 / 2},
$$

and similarly for permutations;

(iii) in all other cases,

$$
(2.1) \lesssim L_{\min }^{1 / 2} N_{\max }^{-1 / 2} \min \left(N_{\max } N_{\min }, L_{\text {med }}\right)^{1 / 2} .
$$


We now prove Proposition 2.1. By duality and the Plancherel theorem, it suffices to show that

$$
\left\|\frac{\left(\xi_{1}+\xi_{2}+\xi_{3}\right)\left\langle\xi_{4}\right\rangle^{s}}{\left\langle\tau_{4}-\omega\left(\xi_{4}\right)+i \xi_{4}^{2}\right\rangle^{1 / 2-\eta} \prod_{j=1}^{3}\left\langle\xi_{j}\right\rangle^{s}\left\langle\tau_{j}-\omega\left(\xi_{j}\right)+i \xi_{j}^{2}\right\rangle^{1 / 2}}\right\|_{[4, \mathbb{R} \times \mathbb{R}]} \lesssim 1 .
$$

We estimate $\left|\xi_{1}+\xi_{2}+\xi_{3}\right|$ by $\left\langle\xi_{4}\right\rangle$. We then apply the inequality

$$
\left\langle\xi_{4}\right\rangle^{s+1} \lesssim\left\langle\xi_{4}\right\rangle^{1 / 2} \sum_{j=1}^{3}\left\langle\xi_{j}\right\rangle^{s+1 / 2}
$$

where we assume that $s>0$. By symmetry it suffices to show that

$$
\left\|\frac{\left\langle\xi_{1}\right\rangle^{-s}\left\langle\xi_{3}\right\rangle^{-s}\left\langle\xi_{2}\right\rangle^{1 / 2}\left\langle\xi_{4}\right\rangle^{1 / 2}}{\left\langle\tau_{4}-\omega\left(\xi_{4}\right)+i \xi_{4}^{2}\right\rangle^{1 / 2-\eta} \prod_{j=1}^{3}\left\langle\tau_{j}-\omega\left(\xi_{j}\right)+i \xi_{j}^{2}\right\rangle^{1 / 2}}\right\|_{[4, \mathbb{R} \times \mathbb{R}]} \lesssim 1 .
$$

We may replace $\left\langle\tau_{2}-\omega\left(\xi_{2}\right)+i \xi_{2}^{2}\right\rangle^{1 / 2}$ by $\left\langle\tau_{2}-\omega\left(\xi_{2}\right)+i \xi_{2}^{2}\right\rangle^{1 / 2-\eta}$. By the $T T^{*}$ identity [12, Lemma 3.7] this estimate is reduced to the bilinear estimate below.

LEMMA 2.3. Let $s>0$; for all $u$, v on $\mathbb{R} \times \mathbb{R}$ and $0<\eta \ll 1$,

$$
\|u v\|_{L^{2}(\mathbb{R} \times \mathbb{R})} \lesssim\|u\|_{X^{1 / 2-\eta,-1 / 2,2}(\mathbb{R} \times \mathbb{R})}\|v\|_{X^{1 / 2, s, 2}(\mathbb{R} \times \mathbb{R})} .
$$

ProOF. By the Plancherel identity, it suffices to show that

$$
\left\|\frac{\left\langle\xi_{1}\right\rangle^{-s}\left\langle\xi_{2}\right\rangle^{1 / 2}}{\left\langle\tau_{1}-\omega\left(\xi_{1}\right)+i \xi_{1}^{2}\right\rangle^{1 / 2}\left\langle\tau_{2}-\omega\left(\xi_{2}\right)+i \xi_{2}^{2}\right\rangle^{1 / 2-\eta}}\right\|_{[3, \mathbb{R} \times \mathbb{R}]} \lesssim 1 .
$$

Observe that, by the translation invariance of the $[k ; Z]$-multiplier norm, we can always restrict our estimate on $\lambda_{j} \gtrsim 1$ and $\max \left(N_{1}, N_{2}, N_{3}\right) \gtrsim 1$. The comparison principle and orthogonality $[12$, Lemmas 3.1, 3.11] reduce our estimate to show that

$$
\begin{aligned}
& \sum_{N_{\max } \sim N_{\operatorname{med}} \sim N} \sum_{L_{1}, L_{2}, L_{3} \gtrsim 1} \frac{\left\langle N_{1}\right\rangle^{-s}\left\langle N_{2}\right\rangle^{1 / 2}}{\max \left(L_{1},\left\langle N_{1}\right\rangle^{2}\right)^{1 / 2} \max \left(L_{2},\left\langle N_{2}\right\rangle^{2}\right)^{1 / 2-\eta}} \\
& \times\left\|X_{N_{1}, N_{2}, N_{3} ; L_{\max } ; L_{1}, L_{2}, L_{3}}\right\|_{[3 ; \mathbb{R} \times \mathbb{R}]} \lesssim 1
\end{aligned}
$$

and

$$
\begin{aligned}
& \sum_{N_{\max } \sim N_{\operatorname{med}} \sim N} \sum_{L_{\max } \sim L_{\operatorname{med}}} \sum_{H \ll L_{\max }} \frac{\left\langle N_{1}\right\rangle^{-s}\left\langle N_{2}\right\rangle^{1 / 2}}{\max \left(L_{1},\left\langle N_{1}\right\rangle^{2}\right)^{1 / 2} \max \left(L_{2},\left\langle N_{2}\right\rangle^{2}\right)^{1 / 2-\eta}} \\
& \times\left\|X_{N_{1}, N_{2}, N_{3} ; H ; L_{1}, L_{2}, L_{3}}\right\|_{[3 ; \mathbb{R} \times \mathbb{R}] \lesssim 1}
\end{aligned}
$$

for all $N \gtrsim 1$. 
First we prove (2.8). We may assume that (2.3) holds. By (2.6), it suffices to prove that

$$
\begin{aligned}
& \sum_{N_{\max } \sim N_{\text {med }} \sim N} \sum_{L_{\text {max }} \sim L_{\operatorname{med}} \gtrsim N_{\min } N_{\max }} L_{\min }^{1 / 2} N_{\text {min }}^{1 / 2} \\
& \times \frac{\left\langle N_{1}\right\rangle^{-s}\left\langle N_{2}\right\rangle^{1 / 2}}{\max \left(L_{1},\left\langle N_{1}\right\rangle^{2}\right)^{1 / 2} \max \left(L_{2},\left\langle N_{2}\right\rangle^{2}\right)^{1 / 2-\eta}} \lesssim 1 .
\end{aligned}
$$

Bounding

$$
\begin{gathered}
\left\langle N_{1}\right\rangle^{-s}\left\langle N_{2}\right\rangle^{1 / 2} \lesssim \frac{N^{1 / 2}}{\left\langle N_{\min }\right\rangle^{s}} \\
\max \left(L_{1},\left\langle N_{1}\right\rangle^{2}\right)^{1 / 2} \max \left(L_{2},\left\langle N_{2}\right\rangle^{2}\right)^{1 / 2-\eta} \gtrsim L_{\min }^{1 / 2} N^{2(1 / 2-\eta)}
\end{gathered}
$$

and performing the $L$ summations, it suffices to show that

$$
\sum_{N_{\text {max }} \sim N_{\text {med }} \sim N} \frac{\left\langle N_{\min }\right\rangle^{1 / 2-s}}{N^{1 / 2-2 \eta}} \lesssim 1,
$$

which is true when $s>0$.

We now prove (2.7). First we assume that (2.4) holds. In this case, we have $N_{1}, N_{2}$, $N_{3} \sim N \gtrsim 1$. Therefore, it suffices to show that

$$
\sum_{L_{\max } \sim N^{2}} \frac{N^{1 / 2-s}}{\max \left(L_{1}, N^{2}\right)^{1 / 2} \max \left(L_{2}, N^{2}\right)^{1 / 2-\eta}} L_{\min }^{1 / 2} L_{\operatorname{med}}^{1 / 4} \lesssim 1,
$$

and this is easily verified when $s>0$ and $L_{\max } \sim N_{\max } N_{\min }$.

Now we consider the case where (2.5) holds. We do not have perfect symmetry and must consider three cases

$$
\begin{array}{ll}
N \sim N_{1} \sim N_{2} \gg N_{3} ; \quad & H \sim L_{3} \gtrsim L_{1}, L_{2}, \\
N \sim N_{2} \sim N_{3} \gg N_{1} ; & H \sim L_{1} \gtrsim L_{2}, L_{3}, \\
N \sim N_{1} \sim N_{3} \gg N_{2} ; & H \sim L_{2} \gtrsim L_{1}, L_{3},
\end{array}
$$

separately.

In the first case we reduce by (2.5) to

$$
\begin{aligned}
& \sum_{N_{3} \ll N} \sum_{1 \lesssim L_{1}, L_{2} \lesssim N N_{3}} \frac{N^{1 / 2-s}}{\max \left(L_{1}, N^{2}\right)^{1 / 2} \max \left(L_{2}, N^{2}\right)^{1 / 2-\eta}} \\
& \times L_{\min }^{1 / 2} N^{-1 / 2} \min \left(N N_{3}, \frac{N}{N_{3}} L_{\text {med }}\right)^{1 / 2} \lesssim 1 .
\end{aligned}
$$

Performing the $N_{3}$ summation, we reduce to

$$
\sum_{1 \lesssim L_{1}, L_{2} \lesssim N^{2}} \frac{N^{1 / 2-s}}{\max \left(L_{1}, N^{2}\right)^{1 / 2} \max \left(L_{2}, N^{2}\right)^{1 / 2-\eta}} L_{\min }^{1 / 2} N^{-1 / 2} N^{1 / 2} L_{\text {med }}^{1 / 4} \lesssim 1,
$$

which is similar to (2.10). 
Considering the second and third cases, it suffices to deal with the worst case

$$
N \sim N_{2} \sim N_{3} \gg N_{1} ; \quad H \sim L_{1} \gtrsim L_{2}, L_{3} .
$$

Using the first part of (2.5),

$$
\sum_{N_{\min } \ll N} \sum_{1 \lesssim L_{\min }, L_{\operatorname{med}} \ll N N_{\min }} \frac{\left\langle N_{\min }\right\rangle^{-s} N^{1 / 2}}{L_{\min }^{1 / 2} N^{2(1 / 2-\eta)}} L_{\min }^{1 / 2} N_{\min }^{1 / 2} \lesssim 1 .
$$

We may assume that $N_{\min } \gtrsim N^{-1}$ since the inner sum vanishes otherwise. Performing the $L$ summation, we reduce to

$$
\sum_{N^{-1} \lesssim N_{\min } \ll N} \frac{\left\langle N_{\min }\right\rangle^{-s} N^{1 / 2} N_{\min }^{1 / 2}}{N^{1-2 \eta}} \lesssim 1,
$$

which holds when $s>0$.

To finish the proof of (2.7), it remains to deal with the case where (2.6) holds. This reduces to

$$
\sum_{N_{\max } \sim N_{\operatorname{med}} \sim N} \sum_{L_{\max } \sim N_{\max } N_{\min }} \frac{\left\langle N_{1}\right\rangle^{-s}\left\langle N_{2}\right\rangle^{1 / 2}}{\max \left(L_{1},\left\langle N_{1}\right\rangle^{2}\right)^{1 / 2} N^{2(1 / 2-\eta)}} L_{\min }^{1 / 2} N^{-1 / 2} L_{\text {med }}^{1 / 2} \lesssim 1 .
$$

Performing the $L$ summations, we reduce to

$$
\sum_{N_{\max } \sim N_{\text {med }} \sim N} \frac{N_{\min }^{1 / 2}}{\left\langle N_{1}\right\rangle^{s} N^{1 / 2-2 \eta}} \lesssim 1,
$$

which is easily verified when $s>0$.

\section{Proof of Theorem $\mathbf{1 . 2}$}

Observing that (1.1) is invariant under the scaling

$$
u(x, t) \rightarrow u_{\lambda}=\frac{1}{\lambda^{1 / 2}} u\left(\frac{x}{\lambda}, \frac{t}{\lambda^{2}}\right), \quad \epsilon \rightarrow \epsilon \frac{1}{\lambda^{1 / 2}}, \quad \phi_{\lambda}=\frac{1}{\lambda^{1 / 2}} \phi\left(\frac{x}{\lambda}\right),
$$

we can see that $\|\phi\|_{L^{2}}$ is invariant under this scaling, and so we require that $\|\phi\|_{L^{2}} \ll 1$. Before embarking on the proof of Theorem 1.2, we establish two results. Let

$$
L(f)(x, t)=W_{0}(t) \psi(t) \int_{\mathbb{R}^{2}} e^{i x \xi} \frac{e^{i t \tau^{\prime}}-e^{-\epsilon|t| \xi^{2}}}{i \tau^{\prime}+\epsilon \xi^{2}} \mathcal{F}\left(W_{0}(-t) f\right)\left(\xi, \tau^{\prime}\right) d \xi d \tau^{\prime} .
$$

Here we take $\psi=\eta_{0}$, and it is easy to verify that

$$
\chi_{\mathbb{R}_{+}}(t) L(f)(x, t)=\chi_{\mathbb{R}_{+}}(t) \psi(t) \int_{0}^{t} W_{\epsilon}(t-\tau) f(\tau) d \tau .
$$

LEMMA 3.1. If $s \geq 1 / 2$ and $\phi \in H^{s}$, there exists $C>0$ such that, for any $0<\epsilon \leq 1$,

$$
\left\|\psi(t) \cdot\left(W_{\epsilon}(t) \phi\right)\right\|_{F^{s}} \leq C\|\phi\|_{H^{s}} .
$$


Proof. We use an idea from [5] in our proof. In view of the definition, it suffices to prove that if $k \in \mathbb{Z}_{+}$, then

$$
\left\|\eta_{k}(\xi) \mathcal{F}\left(\psi(t) \cdot\left(W_{\epsilon}(t) \phi\right)\right)\right\|_{Z_{k}} \leq C\left\|\eta_{k}(\xi) \widehat{\phi}(\xi)\right\|_{L^{2}} .
$$

First, we consider the case $k=0$. Observing that $|\xi| \leq 2$ in this case, and using Taylor's expansion,

$$
\begin{aligned}
\left\|\eta_{0}(\xi) \mathcal{F}\left(\psi(t) W_{\epsilon}(t) \phi\right)\right\|_{X_{0}} \\
\quad \lesssim \sum_{j=0}^{\infty} 2^{j / 2}\left(1+2^{2 j / 5}\right)\left\|\eta_{0}(\xi) \widehat{\phi}(\xi) \mathcal{F}_{t}\left(\psi(t) \sum_{n \geq 0} \frac{(-1)^{n} \epsilon^{n} \xi^{2}}{n !}|t|^{n}\right)(\tau) \eta_{j}(\tau)\right\|_{L_{\xi, \tau}^{2}} \\
\quad \lesssim \sum_{n \geq 0} \frac{4^{n}}{n !}\left\|\eta_{0}(\xi) \widehat{\phi}(\xi)\right\|_{L^{2}}\left\||t|^{n} \psi(t)\right\|_{H^{1}} \\
\quad \lesssim\left\|\eta_{0}(\xi) \widehat{\phi}(\xi)\right\|_{L^{2}},
\end{aligned}
$$

which is (3.4) as desired.

Secondly, we consider the case $k \geq 1$. Observing that if $|\xi| \sim 2^{k}$, then for any $j \geq 0$,

$$
\left\|P_{j}\left(e^{-\epsilon \xi^{2}|t|}\right)(t)\right\|_{L^{2}} \lesssim\left\|P_{j}\left(e^{-\epsilon 2^{2 k}|t|}\right)(t)\right\|_{L^{2}},
$$

which follows from Plancherel's equality and the fact that

$$
\mathcal{F}\left(e^{-|t|}\right)(\tau)=C \frac{1}{1+|\tau|^{2}} .
$$

It follows from the definition that

$$
\begin{aligned}
\left\|\eta_{k}(\xi) \mathcal{F}\left(\psi(t) W_{\epsilon}(t) \phi\right)\right\|_{X_{k}} \\
\quad \lesssim \sum_{j=0}^{\infty} 2^{j / 2} \beta_{k, j}\left\|\eta_{k}(\xi) \widehat{\phi}(\xi) \eta_{j}(\tau) \mathcal{F}_{t}\left(\psi(t) e^{-\epsilon|t| \xi^{2}}\right)(\tau)\right\|_{L_{\xi, \tau}^{2}} \\
\quad \lesssim \sum_{j=0}^{\infty} 2^{j / 2} \beta_{k, j}\left\|\eta_{k}(\xi) \widehat{\phi}(\xi) P_{j}\left(\psi(t) e^{-\epsilon|t| \xi^{2}}\right)(t)\right\|_{L_{\xi, t}^{2}} \\
\quad \lesssim \sum_{j=0}^{\infty} 2^{j / 2} \beta_{k, j}\left\|\eta_{k}(\xi) \widehat{\phi}(\xi)\right\|_{L^{2}} \sup _{|\xi| \sim 2^{k}}\left\|P_{j}\left(\psi(t) e^{-\epsilon|t| \xi^{2}}\right)(t)\right\|_{L_{t}^{2} .}
\end{aligned}
$$

Therefore, it suffices to show that

$$
\sum_{j=0}^{\infty} 2^{j / 2} \beta_{k, j} \sup _{|\xi| \sim 2^{k}}\left\|P_{j}\left(\psi(t) e^{-\epsilon|t| \xi^{2}}\right)(t)\right\|_{L_{t}^{2}} \lesssim 1
$$

We may assume that $j \geq 100$ in the summation. Using the para-product decomposition,

$$
u_{1} u_{2}=\sum_{r=0}^{\infty}\left[\left(P_{r+1} u_{1}\right)\left(P_{\leq r+1} u_{2}\right)+\left(P_{\leq r} u_{1}\right)\left(P_{r+1} u_{2}\right)\right]
$$


and

$$
\begin{aligned}
P_{j}\left(u_{1} u_{2}\right) & =P_{j}\left(\sum_{r \geq j-10}\left[\left(P_{r+1} u_{1}\right)\left(P_{\leq r+1} u_{2}\right)+\left(P_{\leq r} u_{1}\right)\left(P_{r+1} u_{2}\right)\right]\right) \\
& =P_{j}(I+I I) .
\end{aligned}
$$

Now we take $u_{1}=\psi(t)$ and $u_{2}=e^{-\epsilon|t| \xi^{2}}$.

When $j \leq 2 k$, we have $\beta_{k, j} \sim 1$, and the situation can be treated as in [5]. When $j>2 k$, it suffices to bound

$$
\begin{aligned}
\sum_{j \geq 100} & 2^{j / 2} 2^{2(j-2 k) / 5}\left\|P_{j}(I I)\right\|_{L_{\xi}^{\infty} L_{t}^{2}} \\
& \lesssim \sum_{j \geq 100} 2^{j-k} \sum_{r \geq j-10}\left\|P_{r+1} u_{2}\right\|_{L_{\xi}^{\infty} L_{t}^{2}}\left\|P_{\leq r+1} u_{1}\right\|_{L_{\xi, t}^{\infty}} \\
& \lesssim \sum_{j \geq 100} 2^{j-r} \sum_{r \geq j-10} 2^{r-k}\left\|P_{r+1} u_{2}\right\|_{L_{\xi}^{\infty} L_{t}^{2}} \\
& \lesssim \sum_{r} 2^{r-k}\left\|P_{r+1}\left(e^{-\epsilon|t| 2^{2 k}}\right)\right\|_{L_{t}^{2}} \\
& \lesssim 2^{-1-k} \sum_{r} 2^{r+1}\left\|P_{r+1}\left(e^{-\epsilon|t| 2^{2 k}}\right)\right\|_{L_{t}^{2}} \\
& \lesssim 2^{-1-k} \epsilon^{1 / 2} 2^{k}\left\|e^{-|t|}\right\|_{\dot{B}_{2,1}^{1}} \lesssim 1
\end{aligned}
$$

where we use the fact that $e^{-|t|} \in \dot{B}_{2,1}^{1}$ and $\left\|e^{-\epsilon 2^{2 k}|t|}\right\|_{\dot{B}_{2,1}^{1}} \sim \epsilon^{1 / 2} 2^{k}\left\|e^{-|t|}\right\|_{\dot{B}_{2,1}^{1}}$.

The first term, $P_{j}(I)$, in (3.7) can be handled in an easier way. This completes the proof of the proposition.

The next lemma provides an estimate for the retarded linear term.

LEMMA 3.2. For $s \geq 1 / 2$ and $u \in \mathcal{S}(\mathbb{R} \times \mathbb{R})$, there exists $C>0$ such that

$$
\|\psi(t) L(v)\|_{F^{s}} \leq C\|v\|_{N^{s}} .
$$

PROOF. In view of the definitions, it suffices to prove that if $k \in \mathbb{Z}_{+}$, then

$$
\left\|\eta_{k}(\xi) \mathcal{F}(L(v))\right\|_{Z_{k}} \lesssim\left\|\eta_{k}(\xi)(i+\tau-\omega(\xi))^{-1} \mathcal{F}(v)\right\|_{Z_{k}} .
$$

Observe that

$$
\begin{aligned}
\mathcal{F}_{x}(L(v)) & =\psi(t) e^{i t \omega(\xi)} \int_{\mathbb{R}} \frac{e^{i t \tau^{\prime}}-e^{-\epsilon|t| \xi^{2}}}{i \tau^{\prime}+\epsilon \xi^{2}} \widehat{v}\left(\xi, \tau^{\prime}+\omega(\xi)\right) d \tau^{\prime} \\
& =\psi(t) e^{i t \omega(\xi)} \int_{\mathbb{R}} \frac{e^{-i t \omega(\xi)} e^{i t \tau^{\prime}}-e^{-\epsilon|t| \xi^{2}}}{i\left(\tau^{\prime}-\omega(\xi)\right)+\epsilon \xi^{2}} \widehat{v}\left(\xi, \tau^{\prime}\right) d \tau^{\prime} \\
& =\psi(t) \int_{\mathbb{R}} \frac{e^{i t \tau^{\prime}}-e^{i t \omega(\xi)} e^{-\epsilon|t| \xi^{2}}}{i\left(\tau^{\prime}-\omega(\xi)\right)+\epsilon \xi^{2}} \widehat{v}\left(\xi, \tau^{\prime}\right) d \tau^{\prime}
\end{aligned}
$$


and

$$
\mathcal{F}(L(v))(\xi, \tau)=\int_{\mathbb{R}} \frac{\widehat{\psi}\left(\tau-\tau^{\prime}\right)-\mathcal{F}_{t}\left(\psi(t) e^{-\epsilon|t| \xi^{2}}\right)(\tau-\omega(\xi))}{i\left(\tau^{\prime}-\omega(\xi)\right)+\epsilon \xi^{2}} \widehat{v}\left(\xi, \tau^{\prime}\right) d \tau^{\prime} .
$$

For $k \in \mathbb{Z}_{+}$, let $f_{k}\left(\xi, \tau^{\prime}\right)=\mathcal{F}(v)\left(\xi, \tau^{\prime}\right) \eta_{k}(\xi)\left(\tau^{\prime}-\omega(\xi)+i\right)^{-1}$.

For $f_{k} \in Z_{k}$, let

$$
\begin{aligned}
T\left(f_{k}\right)(\xi, \tau)= & \int_{\mathbb{R}} f_{k}\left(\xi, \tau^{\prime}\right) \frac{\widehat{\psi}\left(\tau-\tau^{\prime}\right)-\mathcal{F}_{t}\left(\psi(t) e^{-\epsilon|t| \xi^{2}}\right)(\tau-\omega(\xi))}{i\left(\tau^{\prime}-\omega(\xi)\right)+\epsilon \xi^{2}} \\
& \times\left(\tau^{\prime}-\omega(\xi)+i\right) d \tau^{\prime} .
\end{aligned}
$$

It suffices to show that

$$
\|T\|_{Z_{k} \rightarrow Z_{k}} \leq C \text { uniformly in } k \in Z_{+} .
$$

First, we consider the case $k \in[0,99]$, so $f_{k}=f_{k, j}$ is a function supported in $D_{k, j}$. Let

$$
f_{k, j}^{\#}\left(\xi, \mu^{\prime}\right)=f_{k, j}\left(\xi, \mu^{\prime}+\omega(\xi)\right) \quad \text { and } \quad T^{\#}\left(f_{k, j}\right)(\xi, \mu)=T\left(f_{k, j}\right)(\xi, \mu+\omega(\xi)) .
$$

Thus,

$$
T^{\#}\left(f_{k}\right)(\xi, \tau)=\int_{\mathbb{R}} f_{k}^{\#}\left(\xi, \tau^{\prime}\right) \frac{\widehat{\psi}\left(\tau-\tau^{\prime}\right)-\mathcal{F}_{t}\left(\psi(t) e^{-\epsilon|t| \xi^{2}}\right)(\tau)}{i \tau^{\prime}+\epsilon \xi^{2}}\left(i+\tau^{\prime}\right) d \tau^{\prime} .
$$

Let

$$
w(\tau)=W_{0}(-\tau) v(\tau), \quad k_{\xi}(t)=\psi(t) \int_{\mathbb{R}} \frac{e^{i t \tau^{\prime}}-e^{-\epsilon|t| \xi^{2}}}{i \tau^{\prime}+\epsilon \xi^{2}} \widehat{w}\left(\xi, \tau^{\prime}\right) d \tau^{\prime} .
$$

For (3.9), by definition, it suffices to prove that

$$
\begin{aligned}
& \sum_{j=0}^{\infty} 2^{j / 2} \beta_{k, j}\left\|\eta_{k}(\xi) \eta_{j}(\tau) \mathcal{F}_{t}\left(k_{\xi}\right)(\tau)\right\|_{L_{\xi, \tau}^{2}} \\
& \quad \lesssim \sum_{j=0}^{\infty} 2^{-j / 2} \beta_{k, j}\left\|\eta_{k}(\xi) \eta_{j}(\tau) \widehat{w}(\xi, \tau)\right\|_{L_{\xi, \tau}^{2}} .
\end{aligned}
$$

We use an idea from [5] to decompose

$$
\begin{aligned}
k_{\xi}(t)= & \psi(t) \int_{|\tau| \leq 1} \frac{e^{i t \tau}-1}{i \tau+\epsilon \xi^{2}} \widehat{w}(\xi, \tau) d \tau+\psi(t) \int_{|\tau| \leq 1} \frac{1-e^{-\epsilon|t| \xi^{2}}}{i \tau+\epsilon \xi^{2}} \widehat{w}(\xi, \tau) d \tau \\
& +\psi(t) \int_{|\tau| \geq 1} \frac{e^{i t \tau}}{i \tau+\epsilon \xi^{2}} \widehat{w}(\xi, \tau) d \tau-\psi(t) \int_{|\tau| \geq 1} \frac{e^{-\epsilon|t| \xi^{2}}}{i \tau+\epsilon \xi^{2}} \widehat{w}(\xi, \tau) d \tau \\
= & I+I I+I I I-I V .
\end{aligned}
$$

We estimate each of the above four parts. 
First, we consider the contribution of $I V$. Using the Taylor expansion for $k=0$ and (3.5) for $k \geq 1$, we get

$$
\begin{aligned}
\sum_{j=0}^{\infty} 2^{j / 2} \beta_{k, j}\left\|\eta_{k}(\xi) P_{j}(I V)(t)\right\|_{L_{\xi, t}^{2}} \leq & \sum_{j=0}^{\infty} 2^{j / 2} \beta_{k, j} \int_{|\tau| \geq 1} \frac{\left\|\eta_{k}(\xi) \widehat{w}(\xi, \tau)\right\|_{L_{\xi}^{2}}}{|\tau|} d \tau \\
& \times \sup _{\xi \in I_{k}}\left\|\eta_{k}(\xi) P_{j}\left(\psi(t) e^{-\epsilon|t| \xi^{2}}\right)(t)\right\|_{L_{t}^{2}} \\
\lesssim & \sum_{j=0}^{\infty} 2^{-j / 2} \beta_{k, j}\left\|\eta_{k}(\xi) \eta_{j}(\tau) \widehat{w}(\xi, \tau)\right\|_{L_{\xi, \tau}^{2}}
\end{aligned}
$$

Secondly, we consider the contribution of III. Let

$$
g(\xi, \tau)=\frac{|\widehat{w}(\xi, \tau)|}{\left|i \tau+\epsilon \xi^{2}\right|} \chi_{|\tau| \geq 1} .
$$

When $j>2 k$,

$$
\begin{aligned}
\sum_{j=0}^{\infty} 2^{j / 2} \beta_{k, j}\left\|\eta_{k}(\xi) P_{j}(I I I)(t)\right\|_{L_{\xi, t}^{2}} & \\
& \lesssim \sum_{j=0}^{\infty} 2^{j / 2} 2^{2(j-2 k) / 5}\left\|\eta_{k}(\xi) \eta_{j}(\tau) \widehat{\psi} * g(\xi, \tau)\right\|_{L_{\xi, \tau}^{2}} \\
& \lesssim \sum_{j \geq 1}^{\infty} 2^{9 j / 10} 2^{-4 k / 5}\left\|\frac{\eta_{j}\left(\tau^{\prime}\right)\left\|\eta_{k}(\xi) \widehat{w}\left(\xi, \tau^{\prime}\right)\right\|_{L_{\xi}^{2}}}{\left|i \tau^{\prime}\right|} \chi_{\left|\tau^{\prime}\right| \geq 1}\right\|_{L_{\tau^{\prime}}^{2}} \\
& \lesssim \sum_{j=0}^{\infty} 2^{-j / 2} \beta_{k, j}\left\|\eta_{k}(\xi) \eta_{j}(\tau) \widehat{w}(\xi, \tau)\right\|_{L_{\xi, \tau}^{2}},
\end{aligned}
$$

where we used the fact that $B_{2,1}^{9 / 10}$ is a multiplication algebra and $\mathcal{F}^{-1}(|\widehat{\psi}|) \in B_{2,1}^{9 / 10}$. When $j \leq 2 k$, we can get the desired result by the same estimate as in [5].

Thirdly, we consider the contribution of $I I$. For $\epsilon \xi^{2} \geq 1$, as for $I V$, we get

$$
\begin{aligned}
\sum_{j=0} 2^{j / 2} \beta_{k, j}\left\|\eta_{k}(\xi) P_{j}(I I)(t)\right\|_{L_{\xi, t}^{2}} \lesssim & \sum_{j=0} 2^{j / 2} \beta_{k, j} \int \frac{\|\widehat{w}(\xi, \tau)\|_{L_{\xi}^{2}}}{\langle\tau\rangle} d \tau \\
& \times \sup _{\xi \in I_{k}}\left\|\eta_{k}(\xi) P_{j}\left(\psi\left(1-e^{-\epsilon|t| \xi^{2}}\right)\right)(t)\right\|_{L_{t}^{2}} \\
& \lesssim \sum_{j=0} 2^{-j / 2} \beta_{k, j}\left\|\eta_{k}(\xi) \eta_{j}(\tau) \widehat{w}(\xi, \tau)\right\|_{L_{\xi, \tau}^{2}} .
\end{aligned}
$$


For $\epsilon \xi^{2} \leq 1$, using Taylor's expansion,

$$
\begin{aligned}
& \sum_{j=0} 2^{j / 2} \beta_{k, j}\left\|\eta_{k}(\xi) P_{j}(I I)(t)\right\|_{L_{\xi, t}^{2}} \lesssim \sum_{n \geq 1} \sum_{j=0} 2^{j / 2} \beta_{k, j}\left\|\eta_{k}(\xi) P_{j}\left(|t|^{n} \psi(t)\right) \frac{\epsilon^{n} \xi^{2 n}}{n !} \int_{|\tau| \leq 1} \frac{\widehat{w}(\xi, \tau)}{i \tau+\epsilon \xi^{2}} d \tau\right\|_{L_{\xi, t}^{2}} \\
& \quad \lesssim\left\|\int_{|\tau| \leq 1} \frac{\epsilon \xi^{2}\left|\eta_{k}(\xi) \widehat{w}(\xi, \tau)\right|}{\left|i \tau+\epsilon \xi^{2}\right|} d \tau\right\|_{L_{\xi}^{2}} \\
& \quad \lesssim \sum_{j=0} 2^{-j / 2} \beta_{k, j}\left\|\eta_{k}(\xi) \eta_{j}(\tau) \widehat{w}(\xi, \tau)\right\|_{L_{\xi, \tau}^{2}}
\end{aligned}
$$

where we used the fact that

$$
\left\||t|^{n} \psi(t)\right\|_{B_{2,1}^{9 / 10}} \lesssim\left\||t|^{n} \psi(t)\right\|_{H^{1}} \leq C 2^{n} .
$$

Finally, we consider the contribution of $I$. Using Taylor's expansion,

$$
I=\psi(t) \int_{|\tau| \leq 1} \sum_{n \geq 1} \frac{(i t \tau)^{n}}{n !\left(i \tau+\epsilon \xi^{2}\right)} \widehat{w}(\tau) d \tau
$$

Thus, we get

$$
\begin{aligned}
\sum_{j=0} 2^{j / 2} \beta_{k, j}\left\|\eta_{k}(\xi) P_{j}(I)(t)\right\|_{L_{\xi, t}^{2}} & \\
& \lesssim \sum_{n \geq 1}\left\|\frac{t^{n} \psi(t)}{n !}\right\|_{B_{2,1}^{9 / 10}}\left\|\int_{|\tau| \leq 1} \frac{|\tau|}{\left|i \tau+\epsilon \xi^{2}\right|}\left|\eta_{k}(\xi) \widehat{w}(\xi, \tau)\right| d \tau\right\|_{L_{\xi}^{2}} \\
& \lesssim \sum_{j=0} 2^{-j / 2} \beta_{k, j}\left\|\eta_{k}(\xi) \eta_{j}(\tau) \widehat{w}(\xi, \tau)\right\|_{L_{\xi, \tau}^{2}} .
\end{aligned}
$$

From the definition of the spaces $X_{k}$, we get

$$
\|T\|_{X_{k} \rightarrow X_{k}} \leq C \text { uniformly in } k \geq 1,
$$

as desired.

We now consider $f_{k} \in Y_{k}, k \geq 100$. As in [6], we can assume that $f_{k}$ is supported in the set $\left\{\left(\xi, \tau^{\prime}\right):\left|\tau^{\prime}-\omega(\xi)\right| \leq 2^{k-20}\right\}$. We decompose

$$
g_{k}\left(\xi, \tau^{\prime}\right)=\frac{\tau^{\prime}-\omega(\xi)}{\tau^{\prime}-\omega(\xi)+i} g_{k}\left(\xi, \tau^{\prime}\right)+\frac{i}{\tau^{\prime}-\omega(\xi)+i} g_{k}\left(\xi, \tau^{\prime}\right) .
$$

By (3.12) and the fact that the result

$$
\left\|i\left(\tau^{\prime}-\omega(\xi)+i\right)^{-1} g_{k}\left(\xi, \tau^{\prime}\right)\right\|_{X_{k}} \leq C\left\|g_{k}\right\|_{Y_{k}}
$$


in [6] also holds for our choice $\beta_{k, j}$, it suffices to show that

$$
\begin{aligned}
& \left\|\mathcal{F}_{t}\left(\psi(t) e^{-\epsilon|t| \xi^{2}}\right)(\tau-\omega(\xi)) \int_{\mathbb{R}} g_{k}\left(\xi, \tau^{\prime}\right) \frac{\tau^{\prime}-\omega(\xi)}{\tau^{\prime}-\omega(\xi)+i \epsilon \xi^{2}} d \tau^{\prime}\right\|_{X_{k}} \\
& +\left\|\int_{\mathbb{R}} g_{k}\left(\xi, \tau^{\prime}\right) \frac{\tau^{\prime}-\omega(\xi)}{\tau^{\prime}-\omega(\xi)+i \epsilon \xi^{2}} \widehat{\psi}\left(\tau-\tau^{\prime}\right) d \tau^{\prime}\right\|_{Z_{k}} \leq C\left\|g_{k}\right\|_{Y_{k}} .
\end{aligned}
$$

The first term on the left-hand side of (3.13) can be treated by ideas similar to those in [6]. For the second term, we decompose

$$
g_{k}\left(\xi, \tau^{\prime}\right)=\frac{\tau^{\prime}-\omega(\xi)+i}{\tau^{\prime}-\omega(\xi)+i} g_{k}\left(\xi, \tau^{\prime}\right)+\frac{\tau-\tau^{\prime}}{\tau^{\prime}-\omega(\xi)+i} g_{k}\left(\xi, \tau^{\prime}\right) .
$$

The second term on the left-hand side of (3.13) is dominated by

$$
\begin{aligned}
& C \| \frac{\eta_{[0, k-1]}(\tau-\omega(\xi))}{\tau-\omega(\xi)+i} \int_{\mathbb{R}} g_{k}\left(\xi, \tau^{\prime}\right)\left(\tau^{\prime}-\omega(\xi)+i\right) \\
& \quad \times \widehat{\psi}\left(\tau-\tau^{\prime}\right) \frac{\tau^{\prime}-\omega(\xi)}{\tau^{\prime}-\omega(\xi)+i \epsilon \xi^{2}} d \tau^{\prime} \|_{Y_{k}} \\
& +C \sum_{j \geq k-1} 2^{j / 2} \beta_{k, j} \| \frac{\eta_{j}(\tau-\omega(\xi))}{\tau-\omega(\xi)+i} \int_{\mathbb{R}} g_{k}\left(\xi, \tau^{\prime}\right) \\
& \quad \times \widehat{\psi}\left(\tau-\tau^{\prime}\right) \frac{\tau^{\prime}-\omega(\xi)}{\tau^{\prime}-\omega(\xi)+i \epsilon \xi^{2}} d \tau^{\prime} \|_{L^{2}} \\
& \quad+C \sum_{j \leq k} 2^{j / 2} \| \frac{\eta_{j}(\tau-\omega(\xi))}{\tau-\omega(\xi)+i} \int_{\mathbb{R}} g_{k}\left(\xi, \tau^{\prime}\right) \\
& \quad \times \widehat{\psi}\left(\tau-\tau^{\prime}\right) \frac{\left(\tau-\tau^{\prime}\right)\left(\tau^{\prime}-\omega(\xi)\right)}{\tau^{\prime}-\omega(\xi)+i \epsilon \xi^{2}} d \tau^{\prime} \|_{L^{2}} .
\end{aligned}
$$

This concludes the proof.

We use the following lemma to bound the first term in (3.14); other terms are similarly treated by the method in [6].

LEMMA 3.3. If $k \geq 1,0 \leq j \leq k$ and $g_{k}$ is supported in $I_{k} \times \mathbb{R}$, then

$$
\left\|\mathcal{F}^{-1}\left[\frac{\tau-\omega(\xi)}{\tau-\omega(\xi)+i \epsilon \xi^{2}} \eta_{\leq j}(\tau-\omega(\xi)) g_{k}(\xi, \tau)\right]\right\|_{L_{x}^{1} L_{t}^{2}} \lesssim\left\|\mathcal{F}^{-1}\left[g_{k}(\xi, \tau)\right]\right\|_{L_{x}^{1} L_{t}^{2}}
$$

PROOF. Using Plancherel's theorem, it suffices to prove that

$$
\left\|\int_{\mathbb{R}} e^{i x \xi} \frac{\tau-\omega(\xi)}{\tau-\omega(\xi)+i \epsilon \xi^{2}} \chi_{[k-1, k+1]}(\xi) \eta_{\leq j}(\tau-\omega(\xi)) d \xi\right\|_{L_{x}^{1} L_{\tau}^{\infty}} \leq C .
$$


In proving (3.15), we may assume that $k \geq 100$. Observe that the function on the lefthand side of (3.15) is not zero only if $\tau \approx 2^{2 k}$. By symmetry, it suffices to consider the case $\xi \in\left[2^{k-2}, 2^{k+2}\right]$. Hence we have $\tau-\omega(\xi)=\tau+\xi^{2}$. Changing variable $\tau+\xi^{2}=m$, it suffices to show that

$$
\left|\int_{\mathbb{R}} e^{i x \xi} \frac{m}{m+i \epsilon \xi^{2}} \chi_{[k-1, k+1]}(\xi) \eta_{\leq j}(m) d \xi\right| \leq C .
$$

On twice integrating by parts the left-hand side of (3.16),

$$
\begin{aligned}
& \left|\int_{\mathbb{R}} e^{i x \xi} \frac{m}{m+i \epsilon \xi^{2}} \chi_{[k-1, k+1]}(\xi) \eta_{\leq j}(m) d \xi\right| \\
& =\left|\int_{\mathbb{R}} e^{i x \xi} \frac{m}{m+i \epsilon \xi^{2}} \chi_{[k-1, k+1]}(\xi) \eta_{\leq j}(m) \frac{1}{2 \xi} d m\right| \\
& =\left|\frac{1}{x^{2}} \int_{\mathbb{R}} e^{i x \xi} \frac{d}{d m}\left[\frac{1}{\xi^{\prime}} \frac{d}{d m}\left(\frac{m}{m+i \epsilon \xi^{2}} \chi_{[k-1, k+1]}(\xi) \eta_{\leq j}(m)\right)\right] d m\right|
\end{aligned}
$$

where we use the notation $\xi^{\prime}=d \xi / d m$ and the fact that $\xi^{\prime}=1 / 2 \xi$. To bound the right-hand side of (3.17), it suffices to estimate

$$
\frac{d}{d m}\left[\frac{1}{\xi^{\prime}} \frac{d}{d m}\left(\frac{m}{m+i \epsilon \xi^{2}} \chi_{[k-1, k+1]}(\xi) \eta_{\leq j}(m)\right)\right] .
$$

Let $I=m /\left(m+i \epsilon \xi^{2}\right)$ and $I I=\chi_{[k-1, k+1]}(\xi) \eta_{\leq j}(m)$. It suffices to estimate

$$
\begin{aligned}
& \frac{1}{\xi^{\prime}} I I \frac{d^{2} I}{d m}+\frac{1}{\xi^{\prime}} \frac{d I}{d m} \frac{d I I}{d m}+I \frac{d}{d m}\left(\frac{1}{\xi^{\prime}} \frac{d I I}{d m}\right)+\frac{d I}{d m} \frac{d}{d m}\left(\frac{1}{\xi^{\prime}} I I\right) \\
& =L_{1}+L_{2}+L_{3}+L_{4} .
\end{aligned}
$$

Now we obtain an estimate for $L_{1}$. After some calculation, we obtain

$$
\begin{aligned}
L_{1} & \lesssim \frac{1}{\xi^{\prime}} I I \times \frac{2 i \epsilon m\left(\xi^{\prime}\right)^{2}+2 i \epsilon m \xi \xi^{\prime \prime}+4 i \epsilon m \xi \xi^{\prime}-8 m \epsilon^{2} \xi^{2}\left(\xi^{\prime}\right)^{2}-2 i \epsilon \xi^{2}+4 \epsilon^{2} \xi^{3} \xi^{\prime}}{\left(m+i \epsilon \xi^{2}\right)^{3}} \\
& \lesssim I I
\end{aligned}
$$

where we use the notation $\xi^{\prime \prime}=d^{2} \xi / d m^{2}$ and the fact that $\xi^{\prime \prime}=1 / 4 \xi^{3}$ and $\xi^{\prime}=1 / 2 \xi$. Similarly, for $L_{2}$,

$$
L_{2} \lesssim \frac{1}{\xi^{\prime}} \frac{d I I}{d m} \times \frac{i \epsilon \xi^{2}-2 i \epsilon m \xi \xi^{\prime}}{m+i \epsilon \xi^{2}} \lesssim I I .
$$

Observing the uniform boundedness of $I, d I / d m$, the contributions of $L_{3}$ and $L_{4}$ have been controlled in [6]. 
Collecting the estimates above and noticing the support of $\chi_{[k-1, k+1]}, \eta_{\leq j}(m)$,

$$
\begin{aligned}
\left|\int_{\mathbb{R}} e^{i x \xi} \frac{m}{m+i \epsilon \xi^{2}} \chi_{[k-1, k+1]}(\xi) \eta_{\leq j}(m) d \xi\right| \\
\quad \lesssim\left|\frac{1}{x^{2}} \int_{\mathbb{R}} e^{i x \xi}\left(\frac{1}{2 \xi}+1+\frac{1}{4 \xi^{3}}+2 \xi\right) \chi_{[k-1, k+1]}(\xi) \eta_{\leq j}(m) d m\right| \\
\quad \lesssim\left|\frac{1}{x^{2}} \int_{\mathbb{R}} e^{i x \xi}\left(\frac{1}{2 \xi}+1+\frac{1}{4 \xi^{3}}+2 \xi\right) \chi_{[k-1, k+1]}(\xi) \eta_{\leq j}(\tau-\omega(\xi)) 2 \xi d \xi\right| \\
\quad \lesssim \frac{2^{j-k}}{1+\left(2^{j-k} x\right)^{2}},
\end{aligned}
$$

where we make a change of variable to $m=\tau-\omega(\xi)$. If $\tau=2^{2 k}$, we get the desired result.

For later use, we recall the following trilinear estimate.

Lemma 3.4 [2, Proposition 6.3]. For $s \geq 1 / 2$,

$$
\begin{gathered}
\left\|\partial_{x}\left(\psi(t)^{3} u v w\right)\right\|_{N^{s}} \lesssim\|u\|_{F^{s}}\|v\|_{F^{1 / 2}}\|w\|_{F^{1 / 2}}+\|u\|_{F^{1 / 2}}\|v\|_{F^{s}}\|w\|_{F^{1 / 2}} \\
+\|u\|_{F^{1 / 2}}\|v\|_{F^{1 / 2}}\|w\|_{F^{s}} .
\end{gathered}
$$

REMARK 3.5. In [2], the coefficients are $\beta_{k, j}=1+2^{(j-2 k) / 2}$. Lemma 3.4 also holds for our choice $\beta_{k, j}=1+2^{2(j-2 k) / 5}$; see [4] for details.

Noticing the assumption $\|\phi\|_{L^{2}} \ll 1$ and the scaling (3.1), it suffices to consider (1.1) with data $\phi$ satisfying

$$
\|\phi\|_{H^{s}}=r \ll 1 .
$$

Notice that $F^{s} \subseteq C\left(\mathbb{R} ; H^{s}\right)$ for any $s \geq 0$; see [2]. Collecting (4.3), Lemmas 3.1, 3.2, 3.4 and standard fixed-point machinery, we obtain part (a) of Theorem 1.2. The rest of Theorem 1.2 follows from a standard argument.

\section{Proof of Theorem 1.3}

4.1. Uniform global well-posedness for mBOB. We now extend the local solution obtained above to a global one. We use a conservation law to obtain our goal. From $[8,11]$, we know that there are two conservation laws for the real-valued mBO equation (1.3):

$$
\begin{gathered}
\frac{d}{d t} \int_{\mathbb{R}} u^{2} d x=0, \\
\frac{d}{d t} \int_{\mathbb{R}} \frac{1}{2} u \mathcal{H} u_{x}-\frac{1}{12} u^{4}(x, t) d x=0 .
\end{gathered}
$$

Let $u$ be a smooth solution of (1.1). Multiply by $u$ and use partial integration to obtain

$$
\frac{1}{2}\|u(t)\|_{2}^{2}+\epsilon \int_{0}^{t}\|\Lambda u(\tau)\|_{2}^{2} d \tau=\frac{1}{2}\|\phi\|_{2}^{2},
$$

where we use the notation $\Lambda=\left|\partial_{x}\right|$. 
Turning to the conservation law for (1.1), let

$$
H[u]=\int_{\mathbb{R}} \frac{1}{2} u \mathcal{H} u_{x}-\frac{1}{12} u^{4} d x .
$$

Noticing that (4.2) is a conserved quantity of (1.3),

$$
\begin{aligned}
\frac{d}{d t} H[u] & =\int_{\mathbb{R}} \partial_{t} u \mathcal{H} u_{x}-\frac{1}{3} u^{3} u_{t} d x \\
& =\epsilon \int_{\mathbb{R}} u_{x x} \mathcal{H} u_{x}-\frac{1}{3} u^{3} u_{x x} d x \\
& =-\epsilon\left\|\Lambda^{3 / 2} u\right\|_{2}^{2}+\epsilon \int_{\mathbb{R}} u^{2} u_{x}^{2} d x \\
& \leq-\epsilon\left\|\Lambda^{3 / 2} u\right\|_{2}^{2}+\epsilon\left\|u_{x}\right\|_{2}^{2}\|u\|_{\infty}^{2} \\
& \lesssim-\epsilon\left\|\Lambda^{3 / 2} u\right\|_{2}^{2}+\frac{\epsilon}{2}\left\|\Lambda^{3 / 2} u\right\|_{2}^{2},
\end{aligned}
$$

where we use $\|u\|_{2} \leq\|\phi\|_{2} \ll 1$, the Gagliardo-Nirenberg inequality and the interpolation inequality

$$
\|u\|_{\infty} \lesssim\|u\|_{2}^{1 / 2}\left\|u_{x}\right\|_{2}^{1 / 2}, \quad\left\|u_{x}\right\|_{2} \lesssim\|u\|_{2}^{1 / 3}\left\|\Lambda^{3 / 2} u\right\|_{2}^{2 / 3} .
$$

Therefore,

$$
\sup _{[0, T]}\|u(t)\|_{H^{1 / 2}}+\epsilon^{1 / 2}\left(\int_{0}^{T}\left\|\Lambda^{3 / 2} u(\tau)\right\|_{2}^{2} d \tau\right)^{1 / 2} \leq C\left(T,\|\phi\|_{H^{1 / 2}}\right), \quad \forall T>0 .
$$

Hence the solution is global.

4.2. Limit behaviour. From persistence of regularity of Theorem 1.2, it suffices to show that $s=1 / 2$. We reprise some ideas from $[5,15,16]$ to obtain our result.

Lemma 4.1 [4, Lemma 8.1]. Assume that $\delta>0$. If $s \in \mathbb{R}$ and $u \in L_{t}^{2} H_{x}^{s}$, then

$$
\|u\|_{N^{s}} \lesssim\|u\|_{L_{t}^{2} H_{x}^{s}}
$$

Assume that $u$ is an $H^{1 / 2}$-strong solution of (1.1) obtained above, and that $v$ is an $H^{1 / 2}$-strong solution to (1.3) in [2], with initial data $\phi_{1}, \phi_{2} \in H^{1 / 2}$ respectively. From the scaling (3.1) and the assumption that $\left\|\phi_{i}(x)\right\|_{2} \ll 1, i=1,2$, we may suppose that $\left\|\phi_{1}\right\|_{H^{1 / 2}},\left\|\phi_{2}\right\|_{H^{1 / 2}} \ll 1$. Let $w=u-v$ and $\phi=\phi_{1}-\phi_{2}$. Then $w$ solves

$$
\begin{gathered}
w_{t}+\mathcal{H} w_{x x}-\epsilon w_{x x}=u^{2} u_{x}-v^{2} v_{x}, \quad(x, t) \in \mathbb{R}^{2}, \\
w(0)=\phi(x) .
\end{gathered}
$$

We first view $\epsilon u_{x x}$ as a perturbation to the difference equation of the $\mathrm{mBO}$ equation. Consider the integral equation of (4.5):

$$
w(x, t)=W_{0}(t) \phi-\int_{0}^{t} W_{0}(t-\tau)\left[\epsilon u_{x x}+u^{2} u_{x}-v^{2} v_{x}\right] d \tau, \quad t \geq 0 .
$$


For technical reasons, let

$$
\begin{aligned}
\Phi_{\phi}^{\epsilon}(w(x, t))=\psi(t) & {\left[W_{0}(t) \phi-\epsilon \int_{0}^{t} W_{0}(t-\tau) \psi(\tau) u_{x x}(\tau) d \tau\right.} \\
& \left.-\frac{1}{3} \int_{0}^{t} W_{0}(t-\tau)\left(w\left(v^{2}+u^{2}+v u\right)\right)_{x}(\tau) d \tau\right] .
\end{aligned}
$$

Then $\Phi_{\phi}^{\epsilon}(w)$ solves the integral equation on $t \in[0,1]$. By Lemmas 3.1, 3.2, 3.4 and 4.1,

$$
\begin{array}{r}
\left\|\Phi_{\phi}^{\epsilon}(w)\right\|_{F^{1 / 2}} \lesssim\|\phi\|_{H^{1 / 2}}+\|w\|_{F^{1 / 2}}\|u\|_{F^{1 / 2}}\left(\|v\|_{F^{1 / 2}}+\|u\|_{F^{1 / 2}}\right) \\
+\epsilon\|u\|_{L_{[0,1]}^{2} \dot{H}_{x}^{5 / 2}}+\|w\|_{F^{1 / 2}}\|u\|_{F^{1 / 2}}\|v\|_{F^{1 / 2}}
\end{array}
$$

Since from (3.1) and the assumption that $\left\|\phi_{i}\right\|_{2} \ll 1, i=1,2$,

$$
\|v\|_{F^{1 / 2}} \lesssim\left\|\phi_{2}\right\|_{H^{1 / 2}} \ll 1, \quad\|u\|_{F^{1 / 2}} \lesssim\left\|\phi_{1}\right\|_{H^{1 / 2}} \ll 1
$$

we obtain

$$
\|w\|_{F^{1 / 2}} \lesssim\|\phi\|_{H^{1 / 2}}+\epsilon\|u\|_{L_{[0,1]}^{2} \dot{H}_{x}^{5 / 2}}
$$

From the persistence of regularity of Theorem 1.2, we obtain

$$
\|u-v\|_{C\left([0,1], H^{1 / 2}\right)} \lesssim\left\|\phi_{1}-\phi_{2}\right\|_{H^{1 / 2}}+\epsilon^{1 / 2} C\left(\left\|\phi_{1}\right\|_{H^{5 / 2}},\left\|\phi_{2}\right\|_{H^{1 / 2}}\right) .
$$

For general $\phi_{1} \in H^{5 / 2}, \phi_{2} \in H^{1 / 2}$, using the scaling (3.1), we can show that there exists $T=T\left(\left\|\phi_{1}\right\|_{H^{5 / 2}},\left\|\phi_{2}\right\|_{H^{1 / 2}}\right)>0$ such that

$$
\|u-v\|_{C\left([0, T], H^{1 / 2}\right)} \lesssim\left\|\phi_{1}-\phi_{2}\right\|_{H^{1 / 2}}+\epsilon^{1 / 2} C\left(T,\left\|\phi_{1}\right\|_{H^{5 / 2}},\left\|\phi_{2}\right\|_{H^{1 / 2}}\right) .
$$

Therefore, (4.6) automatically holds for any $T>0$, due to (4.1) and (4.2). Let $S_{T}(\phi)$ be the solution mapping of (1.3) with initial data $\phi$. For fixed $T>0$, we need to prove that for any $\eta>0$, there exists $\sigma>0$ such that if $0<\epsilon<\sigma$, then

$$
\left\|\Phi_{T}^{\epsilon}(\phi)-S_{T}(\phi)\right\|_{C\left([0, T] ; H^{1 / 2}\right)}<\eta .
$$

Denoting $\phi_{K}=P_{\leq K} \phi$, we obtain

$$
\begin{aligned}
\left\|\Phi_{T}^{\epsilon}(\phi)-S_{T}(\phi)\right\|_{C\left([0, T] ; H^{1 / 2}\right)} \leq \| & \Phi_{T}^{\epsilon}(\phi)-\Phi_{T}^{\epsilon}\left(\phi_{K}\right) \|_{C\left([0, T] ; H^{1 / 2}\right)} \\
& +\left\|\Phi_{T}^{\epsilon}\left(\phi_{K}\right)-S_{T}\left(\phi_{K}\right)\right\|_{C\left([0, T] ; H^{1 / 2}\right)} \\
& +\left\|S_{T}\left(\phi_{K}\right)-S_{T}(\phi)\right\|_{C\left([0, T] ; H^{1 / 2}\right)}
\end{aligned}
$$

From Theorem 1.3 and (4.6), we get

$$
\left\|\Phi_{T}^{\epsilon}(\phi)-S_{T}(\phi)\right\|_{C\left([0, T] ; H^{1 / 2}\right)} \lesssim\left\|\phi_{K}-\phi\right\|_{H^{1 / 2}}+\epsilon^{1 / 2} C\left(T, K,\left\|\phi_{K}\right\|_{H^{5 / 2}}\right) .
$$

If we fix $K$ large enough, then let $\epsilon$ go to zero, we get (4.7). 


\section{Acknowledgement}

The authors are grateful to Dr Zihua Guo for helpful discussion and valuable suggestions.

\section{References}

[1] J. Bourgain, 'Fourier transform restriction phenomena for certain lattice subsets and applications to nonlinear evolution equations I, II', Geom. Funct. Anal. 3 (1993), 107-156, 209-262.

[2] Z. Guo, 'Local well-posedness and a priori bounds for the modified Benjamin-Ono equation without using a gauge transformation', arXiv:0807.3764.

[3] Z. Guo, 'Local well-posedness for dispersion generalized Benjamin-Ono equations in Sobolev spaces', arXiv:0812.1825v2.

[4] Z. Guo and B. Wang, 'Global well-posedness and limit behaviour for the modified finite-depthfluid equation', arXiv:0809.2318v1.

[5] Z. Guo and B. Wang, 'Global well-posedness and inviscid limit for the Korteweg-de VriesBurgers equation', J. Differential Equations 246 (2009), 3864-3901.

[6] A. D. Ionescu and C. E. Kenig, 'Global well-posedness of the Benjamin-Ono equation in lowregularity spaces', J. Amer. Math. Soc. 20(3) (2007), 753-798.

[7] C. Kenig, G. Ponce and L. Vega, 'Well-posedness and scattering results for the generalized Korteweg-de Vries equation via the contraction principle', Comm. Pure Appl. Math. 46(4) (1993), $527-620$.

[8] C. Kenig and H. Takaoka, 'Global wellposedness of the modified Benjamin-Ono equation with initial data in $H^{1 / 2}$, Int. Math. Res. Not. (1) (2006), 1-44.

[9] L. Molinet and F. Ribaud, 'On the low regularity of the Korteweg-de Vries-Burgers equation', Int. Math. Res. Not. 37 (2002), 1979-2005.

[10] L. Molinet and F. Ribaud, 'Well-posedness results for the generalized Benjamin-Ono equation with arbitrary large initial data', Int. Math. Res. Not. (70) (2004), 3757-3795.

[11] T. Tao, Nonlinear Dispersive Equations, CBMS Regional Conference Series in Mathematics, 106 (Conference Board of the Mathematical Sciences, Washington, DC, 2006), pp. xvi + 373.

[12] T. Tao, 'Multilinear weighted convolution of $L^{2}$ functions, and applications to nonlinear dispersive equations', Amer. J. Math. 123 (2001), 839-908.

[13] T. Tao, 'Global well-posedness of the Benjamin-Ono equation in $H^{1}(R)^{\prime}, J$. Hyperbolic Differ. Equ. 1 (2004), 27-49.

[14] S. Vento, 'Well-posedness and ill-posedness results for dissipative Benjamin-Ono equations', arXiv:0802.1039v1.

[15] B. Wang, 'The limit behaviour of solutions for the Cauchy problem of the complex GinzburgLandau equation', Comm. Pure Appl. Math. 53 (2002), 0481-0508.

[16] B. Wang and Y. Wang, 'The inviscid limit of the derivative complex Ginzburg-Landau equation', J. Math. Pures Appl. 83 (2004), 477-502.

\section{HUA ZHANG, College of Sciences, North China University of Technology, Beijing 100144, PR China \\ e-mail: zhanghuamaths@163.com}

YUQIN KE, Faculty of Economics, Guangdong University of Business Studies, Guangzhou 510320, PR China

e-mail: keyuqin@gmail.com 